\title{
Operation of an InGrid based X-ray detector at the CAST experiment
}

\author{
Christoph Krieger ${ }^{1}$, ${ }^{\text {, Klaus Desch }}{ }^{1}$, Jochen Kaminski ${ }^{1}$, and Michael Lupberger ${ }^{1}$ \\ ${ }^{1}$ Physikalisches Institut, University of Bonn, Nußallee 12, 53115 Bonn, Germany
}

\begin{abstract}
The CERN Axion Solar Telescope (CAST) is searching for axions and other particles which could be candidates for Dark Matter and even Dark Energy. These particles could be produced in the Sun and detected by a conversion into soft X-ray photons inside a strong magnetic field. In order to increase the sensitivity for physics beyond the Standard Model, detectors with a threshold below $1 \mathrm{keV}$ as well as efficient background rejection methods are required to compensate for low energies and weak couplings resulting in very low detection rates.

Those criteria are fulfilled by a detector utilizing the combination of a pixelized readout chip with an integrated Micromegas stage. These InGrid (Integrated Grid) devices can be build by photolithographic postprocessing techniques, resulting in a close to perfect match of grid and pixels facilitating the detection of single electrons on the chip surface. The high spatial resolution allows for energy determination by simple electron counting as well as for an event-shape based analysis as background rejection method. Tests at an X-ray generator revealed the energy threshold of an InGrid based X-ray detector to be well below the carbon $\mathrm{K}_{\alpha}$ line at $277 \mathrm{eV}$. After the successful demonstration of the detectors key features, the detector was mounted at one of CAST's four detector stations behind an X-ray telescope in 2014. After several months of successful operation without any detector related interruptions, the InGrid based X-ray detector continues data taking at CAST in 2015.

During operation at the experiment, background rates in the order of $10^{-5} \mathrm{keV}^{-1} \mathrm{~cm}^{-2} \mathrm{~s}^{-1}$ have been achieved by application of a likelihood based method discriminating the non-photon background originating mostly from cosmic rays. For continued operation in 2016, an upgraded InGrid based detector is to be installed among other improvements including decoupling and sampling of the signal induced on the grid as well as a veto scintillator to further lower the observed background rates and improving sensitivity.
\end{abstract}

\section{The CAST experiment}

The CERN Axion Solar Telescope (CAST) utilizes a decommissioned LHC prototype dipole magnet to search for weakly interacting particles emerging from the Sun [1]. When the experiment started its search in 2002, the main focus was on the search for axions. Those particles arise from a solution to the strong CP problem via the PecceiQuinn mechanism. As axions provide only weak coupling to ordinary matter as well as a low mass and long lifetime, they are a candidate for at least part of what is called Dark Matter. Axions are produced in the Sun's core through the particle's coupling to photons in the presence of strong electromagnetic fields. To detect axions on the earth, the inverse effect is utilized: Axions can convert back to photons inside a strong magnetic field. As axions originate from the Sun's core, the resulting photons are in the soft $\mathrm{X}$-ray regime of a few $\mathrm{keV}$.

As CAST is able to track the Sun for $2 \times 1.5 \mathrm{~h}$ during sunrise and sunset, four detector stations are equipped with X-ray detectors all providing high sensitivity and low background rates. One of these stations provides an X-ray telescope [2] (a spare from the ABRIXAS space mission, in the following mentioned as the MPE-XRT). Until 2013

\footnotetext{
ae-mail: krieger@physik.uni-bonn.de
}

a pn-CCD detector was mounted behind the X-ray telescope which then needed replacement. Together with the other detectors, mostly Micromegas, it was possible to set the most stringent limits on solar axions up to now [3-5].

When the axion search at CAST was going to finish, CAST started to extend its field of search to other, even more exotic particles with solar origin. Among these is the chameleon [6] which comes along with one of the yet few explanations for what is observed as Dark Energy. The production and detection mechanisms for chameleons are quite similar to those for axions. Chameleons however would not be produced in the Sun's core but in the tachocline which is a region located at 0.7 solar radii and features high differential rotation causing strong magnetic fields. According to the lower temperature in the tachocline compared to the solar core chameleons are produced at much lower energies than axions, resulting in a spectrum peaking below $1 \mathrm{keV}$. Therefore the chameleon search requires detectors with a very low energy threshold in addition to the low background rates. A first glance at CAST's potential in chameleon search has been taken at the end of 2013 using an off the shelf silicon drift detector which was able to set an upper limit on the chameleon photon coupling [7]. 


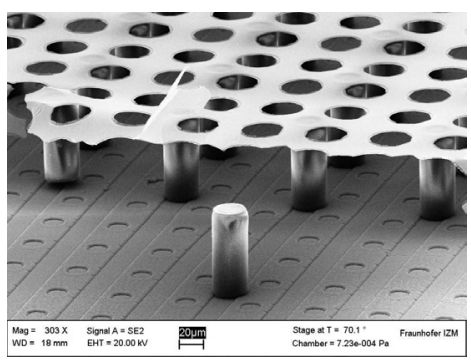

Figure 1: SEM image of an InGrid on top of a Timepix ASIC. Part of the metallic grid has been removed, to show the underlying pixels and pillars. Taken from [13].

\section{Integrated Micromegas}

Among the Micro-Pattern Gaseous Detectors (MPGDs) especially the Micro-MEsh Gaseous Structure detectors (Micromegas) have proven their performance and reliability in many particle physics experiments. Usually, in a Micromegas detector a highly granular gas amplification stage is combined with a rather coarse readout plane, thus, loosing the initial high spatial resolution. As for pad or strip based readout schemes, a granularity in the order of the gas amplification stage's granularity is unfeasible due to the high channel density and necessity to have the electronic readout chain routed to each pad or strip, the only way is to combine a Micromegas-like gas amplification stage with highly integrated readout electronics as it can be found in pixelized readout chips.

To avoid misalignment and the resulting Moire patterns, it is critical to have a close to perfect match between pixels and holes in the Micromegas' mesh. This is achieved by application of modern photolithographic postprocessing techniques, which allow to build up a Micromegas-like structure directly on top of a pixelized readout chip. A reasonable choice for the pixelized readout chip is the Timepix ASIC [8], which provides many useful features for the application in an MPGD, e.g. a Time Projection Chamber (TPC). The Timepix ASIC offers $256 \times 256$ pixels on $2 \mathrm{~cm}^{2}$ of active surface with a pixel pitch of $55 \mu \mathrm{m}$. Each pixel contains its own preamplifier, discriminator and counting logic allowing for either measurement of the arriving charge or the time the discriminator threshold was surpassed.

The production of these integrated Micromegas (also called InGrids, derived from Integrated Grid) devices was first pioneered at the University of Twente [9, 10] and is nowadays done at the IZM in Berlin [11] allowing for the processing of full eight inch Timepix wafers and thus, fulfilling the demands of R\&D regarding the number of InGrids available at a time. Figure 1 shows an SEM image of an InGrid structure made at the IZM in Berlin. To protect the pixels' electronics from discharges, a thin (typically 4 or $8 \mu \mathrm{m}$ ) resistive layer made from silicon nitride [12] is deposited on the chip's surface before building up the Micromegas structure. This protection layer does not prevent discharges but spreads the arriving charge over many pixels in case of spark happening.

\section{The InGrid based X-ray detector}

An X-ray detector based on the InGrid technology, treated in the previous section, has been build. After successful tests with a first prototype [14] the detector version to be installed at the CAST experiment was designed basing on the CAST (sunset) Micromegas detectors [15]. An exploded view of the detector can be found in figure 2 . The Timepix ASIC with its InGrid stage on top is the central element of the detector housed in the exchangeable readout module which is read out by an FPGA based readout system developed at Bonn [13]. To reduce field distortions at the chip's borders, it is covered by a field shaping electrode with a cutout just the size of the chip's active area to which the electric potential according to its position within the drift field is applied. The drift region of the detector is defined by a ring made of acrylic glass closed by the cathode frame made from copper covered with a film of $2 \mu \mathrm{m}$ metalized Mylar.

To allow very low energetic X-ray photons to enter the detector volume, the copper is removed on a 16 by $16 \mathrm{~mm}^{2}$ area, small ribs have been kept to provide a strongback for the thin window below. At CAST, the detector needs to be connected to the vacuum of the MPE-XRT, thus, requiring the window to withstand a pressure difference of 1050 mbar and not leaking too much of the detectors gas filling into the vacuum. The $2 \mu \mathrm{m}$ Mylar film has been chosen as a good compromise between robustness and transparency. Similarly, the gas used to operate the detector, a mixture of Argon and isobutane $\left(\mathrm{Ar} / \mathrm{iC}_{4} \mathrm{H}_{10}\right.$ 97.7/2.3), was chosen as a compromise between gas amplification properties and the wish to avoid the usage of gas mixtures regarded as flammable.

An X-ray photon entering the detector will likely hit a gas atom and produce a bunch of primary electrons through ionization. The number of primary electrons depends strongly on the energy of the initial photon. Due to the electric field between cathode and InGrid, the electrons

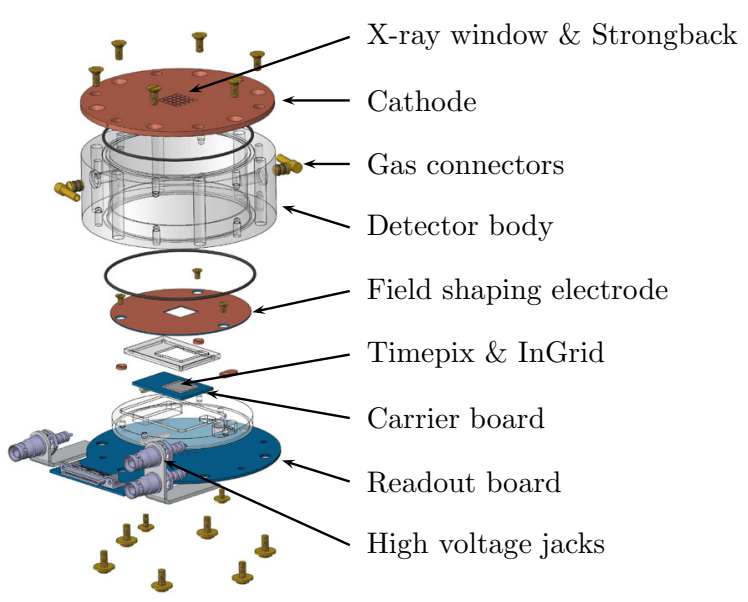

Figure 2: An exploded view of the InGrid based X-ray detector including labeling of main parts. Taken from [16]. 


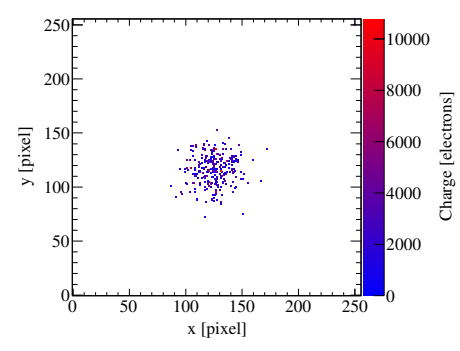

Figure 3: Cloud of primary electrons originating from an $8 \mathrm{keV}$ X-ray photon, recorded with an InGrid based X-ray detector. Depicted area is the complete active area of a Timepix ASIC. Charge resulting from gas amplification above each pixel is color coded on the $\mathrm{z}$-axis.

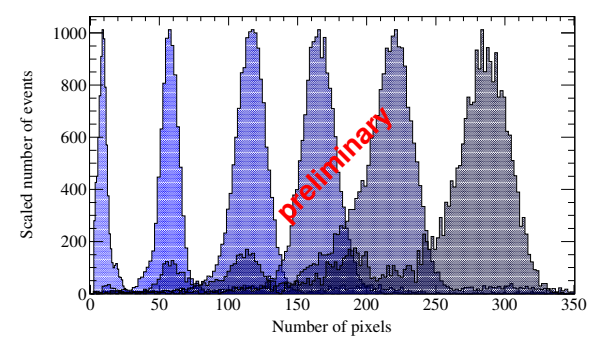

Figure 4: Characteristic X-ray lines recorded during tests at an X-ray generator, each line was recorded separately, all spectra have been normalized to the same height. From left to right: $277 \mathrm{eV}, 1.5 \mathrm{keV}, 3 \mathrm{keV}, 4.5 \mathrm{keV}, 5.9 \mathrm{keV}$ and $8 \mathrm{keV}$.

will drift towards the gas amplification structure, diffusing thereby. This results in an electron cloud of almost circular shape being detected on the readout chip. Due to the high granularity of Timepix ASIC and InGrid primary electrons can be identified as pixels hit, if they are seperated by more than the pixel pitch of $55 \mu \mathrm{m}$ at the level of the grid. This allows for energy measurement based on simple pixel counting as well as for an event-shape-based discrimination of non-photon events. A sample X-ray event is shown in figure 3. The energy threshold of this kind of detector is given by the energy needed to create one primary electron, typically about $25 \mathrm{eV}$. To verify the ability to detect very low energetic X-ray photons (below $1 \mathrm{keV}$ ), the detector was tested at an X- ray generator at CERN [16] prior to the installation at the CAST experiment. These tests successfully demonstrated the ability to detect X-ray photons down to energies as low as the carbon $\mathrm{K}_{\alpha}$ line at $277 \mathrm{eV}$, see figure 4 for a set of characteristic $\mathrm{X}$-ray lines recorded during the tests.

\section{Installation behind the MPE-XRT}

To install the InGrid based X-ray detector behind the MPE-XRT at the CAST experiment, a small vacuum system had to be set up connecting the gas filled detector to

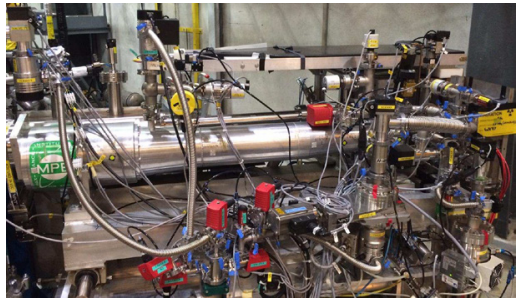

(a)

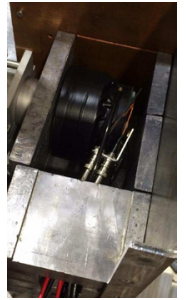

(b)
Figure 5: Pictures of the setup for the InGrid based X-ray detector at the CAST experiment: (a) shows the MPE$\mathrm{XRT}$, its vacuum system including the part allowing to connect the detector which is housed in a lead shielding on the right; (b) shows the detector within its partially assembled lead shielding.

the good vacuum of the telescope. To be able to reach vacuum pressures low enough so the gate valves to the telescope can be opened a differential pumping scheme was applied using a thin differential window made from $0.9 \mu \mathrm{m}$ Mylar. This vacuum system along with the infrastructure for the detector itself was installed at CAST in 2014. To allow for calibration of the detector while taking data at the experiment, an ${ }^{55} \mathrm{Fe}$ source can be moved in front of the detector inside the vacuum. The vacuum system of the InGrid based detector and of the MPE-XRT is illustrated in figure 5a. From October 2014 until November 2014, the detector took first data while tracking the Sun. Data taking continued in 2015 for several months until the detector was dismounted end of November 2015.

To reduce background events induced by cosmic rays, the detector is shielded within a small enclosure made from low activity lead provided by the University of Zaragoza. Figure 5b shows the detector inside the lead shielding with only the top layer of lead bricks missing.

\section{Background reduction \& measured rates}

The Timepix ASIC of the InGrid based X-ray detector is operated untriggered and is read out framewise, each frame is taken with an exposure time of $0.98 \mathrm{~s}$, which is reasonable as the expected event rate is low. About $90 \%$ of the frames recorded during a background run are empty. Reconstruction of the events is performed utilizing a modified clustering algorithm. From the resulting reconstructed events, only those which were most likely caused by an X-ray photon are of interest. To separate those from the non-photon-like events, a likelihood based method is applied. The likelihood is calculated from the distributions of three event-shape variables taking advantage of the Timepix ASIC's and InGrid's high granularity. The variables are excentricity, length divided by transverse rms and fraction of pixels within a radius of one transverse rms. These variables have been chosen in a way so they do not depend on gas parameters like temperature. The reference distributions are taken from the tests at the X-ray 


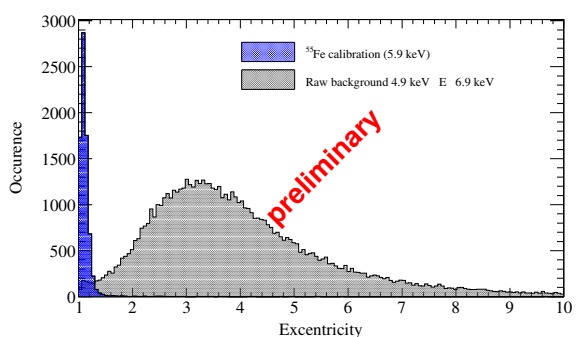

Figure 6: Excentricity distribution for $5.9 \mathrm{keV}$ photons from an ${ }^{55} \mathrm{Fe}$ source and for raw background events of comparable energy. A clear separation is visible, only few of the raw background events look photon-like.

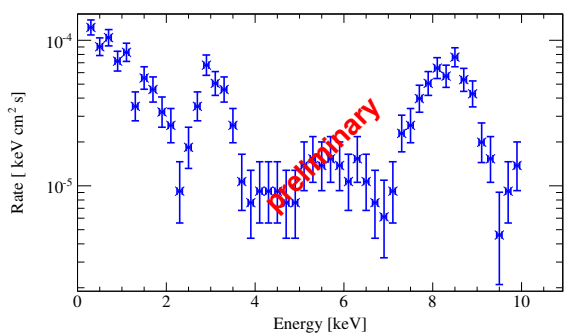

Figure 7: Background spectrum obtained with the InGrid based X-ray detector operated at the CAST experiment. Rate is calculated from events within the innermost 5 by $5 \mathrm{~mm}^{2}$ area of the chip.

generator, the reference dataset to be used is selected according to the energy of the reconstructed events. The cut value for the likelihood is chosen so that for all energy ranges a software efficiency of $80 \%$ is maintained. Figure 6 shows the distribution of the excentricity for raw background events and a calibration dataset taken under usage of an ${ }^{55} \mathrm{Fe}$ source. Only a small fraction of the raw background events appears to be photon-like.

When applying the likelihood based method to reduce the background by only selecting photon-like events the background spectrum shown in figure 7 is obtained which shows the rate within the innermost 5 by $5 \mathrm{~mm}^{2}$ area of the chip, where lowest background rates are achieved. For energies above $2 \mathrm{keV}$, a background rate of a few $10^{-5} \mathrm{keV}^{-1} \mathrm{~cm}^{-2} \mathrm{~s}^{-1}$ is achieved except for two peaks at $3 \mathrm{keV}$ and $8 \mathrm{keV}$. While the latter one originates from florescence photons created by cosmic rays traversing the copper cathode, the other one is caused by florescence photons from the Argon gas in the detector. Additionally, cosmic tracks perpendicular to the chip's surface can mimic X-ray-like events, which also contribute to the peak at about $8 \mathrm{keV}$. For energies below $2 \mathrm{keV}$, the background rate rises as the likelihood method's efficiency decreases due to blurring of the reference distributions. This is caused by the reduced number of activated pixels for low energy events, increasing the spread for the event-shape variables. Still, the background rate below $2 \mathrm{keV}$ with at maximum $10^{-4} \mathrm{keV}^{-1} \mathrm{~cm}^{-2} \mathrm{~s}^{-1}$ is one order of magnitude lower than for the silicon drift detector used for CAST's first look at chameleons [7] and should therefore allow for an improved limit on the chameleon couplings in case of absence of a signal.

\section{Summary \& Outlook}

An X-ray detector based on integrated Micromegas technology utilizing a pixelized readout chip has been successfully build, tested and installed at the CAST experiment. It was operated from October 2014 until end of November 2015 and was able to achieve background rates down to a few $10^{-5} \mathrm{keV}^{-1} \mathrm{~cm}^{-2} \mathrm{~s}^{-1}$ above $2 \mathrm{keV}$ and at maximum $10^{-4} \mathrm{keV}^{-1} \mathrm{~cm}^{-2} \mathrm{~s}^{-1}$ below.

For 2016, an upgraded version of the InGrid based Xray detector is to be installed and operated at the CAST experiment. Upgrades will include decoupling and sampling of the signal induced on the InGrid's grid, integration of one or more veto scintillators, both aiming to further reduce the background levels by providing a better handle to discriminate events caused by cosmic rays. Additionally, an ultra thin (few hundred $\mathrm{nm}$ ) detector window made of silicon nitride on a silicon and ceramic strongback is foreseen to improve the detector's sensitivity by increasing Xray transmission of the entrance window.

\section{References}

[1] K. Zioutas et al., Nucl. Instr. Meth. Phys. Res. A 425, 480 (1999)

[2] M. Kuster et al., New J. Phys. 9, 169 (2007)

[3] E. Arik et al., J. Cosmol. Astropart. Phys. 2009, 008 (2009)

[4] M. Arik et al., Phys. Rev. Lett. 112, 091302 (2014)

[5] M. Arik et al., Phys. Rev. D 92, 021101 (2015)

[6] P. Brax et al., Phys. Rev. D 85, 043014 (2012)

[7] V. Anastassopoulo et al., Phys. Lett. B 749, 172 (2015)

[8] X. Llopart et al., Nucl. Instr. Meth. Phys. Res. A 581, 485 (2007)

[9] M. Chefdeville et al., Nucl. Instr. Meth. Phys. Res. A 556, 490 (2006)

[10] H. van der Graaf, Nucl. Instr. Meth. Phys. Res. A 580, 1023 (2007)

[11] M. Lupberger et al., InGrid: Pixelated Micromegas detectors for a Pixel-TPC, in [17]

[12] Y. Bilevych et al., Nucl. Instr. Meth. Phys. Res. A 629, 66 (2011)

[13] M. Lupberger, J. Instrum. 5, C01033 (2014)

[14] C. Krieger et al., Nucl. Instr. Meth. Phys. Res. A 729, 905 (2013)

[15] J. Galán et al., J. Instrum. 5, P01009 (2010)

[16] C. Krieger et al., An InGrid based Low Energy X-ray Detector for the CAST Experiment, in [17]

[17] Proceedings of the 3rd International Conference on Technolgy and Instrumentation in Particle Physics (PoS, 2014) 